\title{
Could vitamin D and bicarbonate supplementation synergize to mitigate age-related loss of muscle?
}

\author{
D. Travis Thomas
}

Received: 18 June 2013/Accepted: 25 June 2013/Published online: 4 July 2013

(C) Springer Science+Business Media New York 2013

In patients with kidney disease receiving hemodialysis, metabolic acidosis is closely tied to muscle pathology and is known to exacerbate age-related loss of muscle mass and function. To a lesser extent, the "Western" diet is thought to contribute to mild (sub-clinical) metabolic acidosis via the supply of acid precursors from foods [1], but the clinical significance to chronic age-related muscle degeneration has received little attention. This may partly be due to equivocal data on the benefits of neutralizing diet-induced metabolic acidosis for improving muscle-related outcomes in patients without kidney disease [2]. Despite limited data, acidosis has been shown to stimulate muscle proteolysis and is associated with negative functional outcomes including reduced peak torque and lower gait speed [3]. This is important to note because aged adults are less able to excrete excess $\mathrm{H}^{+}$ions and are more prone to develop mild, but slowly increasing metabolic acidosis that may be exacerbated by a "Western" diet [1]. Furthermore, approximately one out of four adults older than 50 years of age have low serum bicarbonate levels to neutralize an acidogenic diet. Because of these factors, recommendations for future work include examining the effects of alkali therapy on functional outcomes, nitrogen balance, strength and other relevant measures of physical function, in aged healthy adults [3].

Vitamin D status (measured as $25(\mathrm{OH}) \mathrm{D}$ ) is also garnering interest as a player in preserving skeletal muscle mass and function in aging. Although, the study of vitamin $\mathrm{D}$ deficiency and repletion, in relation to muscle metabolic function in aging is relatively new, vitamin D deficiency

D. Travis Thomas $(\bowtie)$

Division of Clinical Nutrition, University of Kentucky, Lexington, KY, USA

e-mail: david.t.thomas@uky.edu has already been associated with several negative muscle outcomes including muscle degeneration with fat infiltration [4]. Loss of muscle fiber size and reduction in muscle protein synthesis have also been documented and is thought to be clinically significant since vitamin D deficiency is considered as a worldwide epidemic [5].

In this issue of Endocrine, Ceglia et al. [6] investigated the interactions between potassium bicarbonate and vitamin D supplementation to shed light on the underlying mechanisms associated with muscle atrophy, conservation, and fiber morphology in male rats. The author's rationale for examining the vitamin D/bicarbonate interaction was based on research from the 1970s citing that depletion of vitamin $\mathrm{D}$ resulted in metabolic acidosis, whereas repletion resulted in a metabolic alkalosis by changes in renal tubule bicarbonate reabsorption. An additional cited study provided support that acute metabolic acidosis resulted in suppression of 1-alpha hydroxylase activity, collectively suggesting that the alterations in either vitamin D status or acid-base balance may affect the other and, thus, have detrimental impact on muscle tissue [6]. Ceglia et al. [6] found that potassium bicarbonate supplementation resulted in $35 \%$ lower urinary nitrogen to creatinine $(\mathrm{UNi} / \mathrm{Cr})$ ratio independent of vitamin D status. In vitamin D deficient rats, potassium bicarbonate resulted in $28 \%$ increase in $\mathrm{UNi} / \mathrm{Cr}$ compared to rats with normal vitamin D levels, but the findings did not reach the statistical significance. Furthermore, in rats experiencing metabolic acidosis, higher vitamin D status seemed to work synergistically with bicarbonate to potentiate Akt activation, a protein kinase involved in several anabolic pathways. As a result of their findings, the authors suggested that longer more comprehensive investigation is required to advance and confirm the impact of a dietary intervention on ubiquitin-proteasome pathway at different pathway time points and to fully 
characterize the effects of bicarbonate and vitamin D repletion on human muscle morphology [6].

The primary contribution of this study was that rats with higher vitamin D status trended toward losing less urinary nitrogen and experienced greater Akt activation. Alterations in vitamin D status appeared to modify the alkali effect despite data that Akt expression and several upstream and downstream targets did not significantly change. This study suggested that sufficient vitamin $\mathrm{D}$ conditions may be necessary to enhance the Akt activation and to augment the benefits of bicarbonate supplementation. The fact that Ceglia et al. [6] did not see vitamin D-dependent type 1 or 2 muscle fiber effects may have been due to the short length of the study and rats not reaching sufficiently low serum $25(\mathrm{OH}) \mathrm{D}$ levels prior to repletion. In support of this study, vitamin $\mathrm{D}$ and bicarbonate supplementation both appear to be associated with muscle functional performance in older adults [7, 8], and vitamin D repletion in both rat and human models support type 2 fiber alterations/regeneration [5]. A better understanding of the interaction between acid-base balance and vitamin D status for improving muscle mass and function are needed, and novel findings by Ceglia et al. [6] provide support for investigating this interaction in aging human muscle.

The authors should be commended on their scientific approach of combining bicarbonate and vitamin D treatment. The integration of promising interventions combined with vitamin $\mathrm{D}$ repletion may provide novel insight and support for vitamin $\mathrm{D}$ as a synergistic player in muscle metabolic pathways and inform future "comparative effectiveness" studies. For example, vitamin D receptor expression in skeletal muscle has been associated with muscle regeneration and repair [9] suggesting a synergistic interaction between exercise and vitamin D. Reversal of vitamin D depletion "combined" with other promising interventions (e.g., exercise, bicarbonate supplementation) may provide the most impact for preserving or improving muscle mass and function in aging.

Despite the novel design of this study, little insight was provided in the discussion on translating these findings to future directions for applied aging research in humans. Is alkaline treatment achievable "and" practical with diet manipulation alone? Can age-associated muscle performance decline be delayed with chronic potassium bicarbonate supplementation? Given normal age-associated decline in renal function coupled with some reports of bicarbonate intolerance, is safety a significant barrier to long-term use of potassium bicarbonate? The study also did not address how their administration of potassium bicarbonate translates to human outcomes or how study limitations could be addressed to advance human trials. Although, there is some evidence that alkaline salts have a favorable acute effect on muscle mass, questionable clinical impact related to clinical trial feasibility preclude specific recommendations that need to be elucidated in future clinical trials. Ceglia et al. [6] findings ultimately need to be confirmed in human interventions that address significant design barriers and inherent challenges related to: reliability and variability of urine measures (protein, glucose, and ketones), diagnosis of hypertension, use of diuretic medications, sex differences, self-reported dietary intake, body mass index, alkaline dosing, and physical activity level [1]. Controlling for renal function is an additional challenge, as age-related decline is known to increase blood $\mathrm{H}^{+}$ions, which could inappropriately reflect diet in older individuals. Until these studies take place, available human evidence to support the clinical relevance and need for reversing sub-clinical metabolic acidosis seems limited [1].

Inspite of the aforementioned limitations, future human work in this area is warranted to prospectively examine the effect alkalization has on muscle mass and function in aging adults and how vitamin $\mathrm{D}$ repletion works to mediate these effects. The most recent human study related to these findings was by Dawson-Hughes et al. [8] who provided bicarbonate for 3 months in middle-aged adults. The authors reported a significant decreased net acid excretion and modestly improved lower extremity peak muscle power, endurance and a higher reduction in nitrogen excretion in healthy older women compared to men. The authors suggested that bicarbonate may offer a low-cost intervention that may attenuate age-related loss of muscle performance and mass in elderly. The vitamin D interaction to acidosis also needs further clarification. For example, animal data cited by Ceglia et al. [6] reporting that acidosis interferes with the adaptive enzymatic control of 25-hydroxycholecalciferol metabolism in the vitamin D depleted state needs to be replicated in humans.

In summary, efforts to mitigate age-related muscle wasting, loss of muscle function, and exercise capacity continue to be a focus of scientific inquiry and this study provides some evidence that bicarbonate and vitamin D supplementation may play a beneficial synergistic role. Along with the potential muscle benefits obtained by chronic reversal of mild acidosis, evidence is accumulating that vitamin D may enhance the muscle metabolic function and positively augment the effects of bicarbonate supplementation. It is possible that one undervalued aspect of vitamin D repletion is its ability to enhance other metabolic interventions and pathways to preserve muscle mass and function in aging. Until future trials work to elucidate practical bicarbonate-based interventions in aged humans and expand our knowledge of the role vitamin D plays both as an independent factor and adjuvant to other therapies, it seems prudent to continue to recommend diets high in fruits and vegetables as a strategy to limit acidosis while 
being proactive in the prevention of vitamin D insufficiency.

\section{References}

1. A.A. Welch, A. Mulligan, S.A. Bingham, K.T. Khaw, Urine pH is an indicator of dietary acid-base load, fruit and vegetables and meat intakes: results from the European Prospective Investigation into Cancer and Nutrition (EPIC)-Norfolk population study. Br. J. Nutr. 99(6), 1335-1343 (2008)

2. J. Pizzorno, L.A. Frassetto, J. Katzinger, Diet-induced acidosis: is it real and clinically relevant? Br. J. Nutr. 103(8), 1185-1194 (2010)

3. M.K. Abramowitz, T.H. Hostetter, M.L. Melamed, Association of serum bicarbonate levels with gait speed and quadriceps strength in older adults. Am. J. kidney. dis. 58(1), 29-38 (2011)

4. V. Gilsanz, A. Kremer, A.O. Mo, T.A. Wren, R. Kremer, Vitamin $\mathrm{D}$ status and its relation to muscle mass and muscle fat in young women. J. Clin. Endocrinol. metab. 95(4), 1595-1601 (2010)
5. B. Hamilton, Vitamin D and human skeletal muscle. Scand. J. Med. Sci. Sports. 20(2), 182-190 (2010)

6. L. Ceglia, D.A. Rivas, R.M. Pojednic, L.L. Price, S.S. Harris, D. Smith, R.A. Fielding, B. Dawson-Hughes, Effects of alkali supplementation and vitamin D insufficiency on rat skeletal muscle. Endocrine (2013). doi:10.1007/s12020-013-9976-0

7. H.A. Bischoff-Ferrari, T. Dietrich, E.J. Orav, F.B. Hu, Y. Zhang, E.W. Karlson, B. Dawson-Hughes, Higher 25-hydroxyvitamin D concentrations are associated with better lower-extremity function in both active and inactive persons aged $>$ or $=60 \mathrm{y}$. Am. J. Clin. Nutr. 80(3), 752-758 (2004)

8. B. Dawson-Hughes, C. Castaneda-Sceppa, S.S. Harris, N.J. Palermo, G. Cloutier, L. Ceglia, G.E. Dallal, Impact of supplementation with bicarbonate on lower-extremity muscle performance in older men and women. Osteoporos. Int. 21(7), 1171-1179 (2010)

9. R. Srikuea, X. Zhang, O.K. Park-Sarge, K.A. Esser, VDR and CYP27B1 are expressed in $\mathrm{C} 2 \mathrm{C} 12$ cells and regenerating skeletal muscle: potential role in suppression of myoblast proliferation. Am. J. Physiol. Cell Physiol. 303(4), C396-405 (2012). doi: 10.1152/ajpcell.00014.2012 